\section{Quantitative ultrasound radiofrequency data analysis for the assessment of hepatic steatosis using the controlled attenuation parameter as a reference standard}

\author{
Sun Kyung Jeon', Ijin Joo', So Yeon Kim², Jong Keon Jang ${ }^{2}$, Juil Park', Hee Sun Park', \\ Eun Sun Lee ${ }^{4}$, Jeong Min Lee ${ }^{1}$ \\ 'Department of Radiology, Seoul National University Hospital, Seoul National University \\ College of Medicine, Seoul; '2Department of Radiology and Research Institute of Radiology, \\ Asan Medical Center, University of Ulsan College of Medicine, Seoul; ${ }^{3}$ Department of \\ Radiology, Konkuk University School of Medicine, Seoul; ${ }^{4}$ Department of Radiology, Chung- \\ Ang University Hospital, Chung-Ang University College of Medicine, Seoul, Korea
}

Purpose: This study was aimed to investigate the value of quantitative ultrasound (US) parameters from radiofrequency (RF) data analysis for assessing hepatic steatosis, using controlled attenuation parameter (CAP)-based steatosis grades as the reference standard.

Methods: We analyzed 243 participants with both B-mode liver US with RF data acquisition and CAP measurements. On B-mode US images, hepatic steatosis was visually scored (0/1/2/3, none/mild/moderate/severe), and the hepatorenal index (HRI) was calculated. From the RF data analysis, the tissue scatter-distribution imaging parameter (TSI-p) and tissue attenuation imaging parameter (TAI-p) of the liver parenchyma were measured. US parameters were correlated with CAP-based steatosis grades (S0/1/2/3, none/mild/moderate/severe) and their diagnostic performance was evaluated using receiver operating characteristic (ROC) curve analysis. Multivariate linear regression analysis was performed to identify determinants of TSI-p and TAI-p. Results: Participants were classified as having SO $(n=152), S 1(n=54), S 2(n=14)$, and S3 ( $n=23)$ on CAP measurements. TSI- $p$ and TAI- $p$ were significantly correlated with steatosis grades ( $\rho$ $=0.593$ and $\rho=-0.617, P<0.001$ for both). For predicting $\geq S 1, \geq S 2$, and $S 3$, the areas under the ROC curves (AUCs) of TSI-p were 0.827/0.914/0.917; TAI-p, 0.844/0.914/0.909; visual scores, 0.659/0.778/0.794; and HRI, 0.629/0.751/0.759, respectively. TSI-p and TAI-p had significantly higher AUCs than did visual scores or $\mathrm{HRI}$ for $\geq \mathrm{S} 1$ or $\geq \mathrm{S} 2$ ( $\mathrm{P} \leq 0.003)$. In the multivariate analysis, the transient elastography-based fibrosis grade $(P=0.034)$ and steatosis grade $(P<0.001)$ were independent determinants of TSI-p, while steatosis grade $(P<0.001)$ was an independent determinant of TAI-p.

Conclusion: TSI-p and TAI-p derived from US RF data may be useful for detecting hepatic steatosis and assessing its severity.

Keywords: Ultrasonography; Liver; Fatty liver

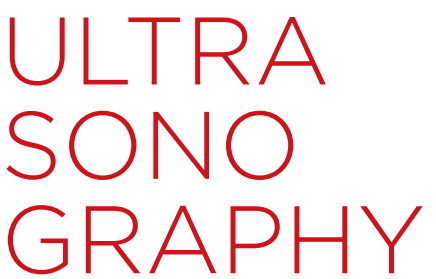

ORIGINAL ARTICLE

https://doi.org/10.14366/usg.20042 pISSN: 2288-5919 - elSSN: 2288-5943 Ultrasonography 2021;40:136-146

Received: March 19, 2020

Revised: May 8, 2020

Accepted: May 9, 2020

Correspondence to: ljin Joo, MD, Department of Radiology, Seoul National University Hospital, Seoul National University College of Medicine, 101 Daehak-ro, Jongno-gu, Seoul 03080, Korea

Tel. +82-2-2072-3107

Fax. +82-2-743-6385

E-mail: hijijin@gmail.com

This is an Open Access article distributed under the terms of the Creative Commons Attribution NonCommercial License (http://creativecommons.org/ licenses/by-nc/4.0/) which permits unrestricted noncommercial use, distribution, and reproduction in any medium, provided the original work is properly cited.

Copyright (C) 2021 Korean Society of Ultrasound in Medicine (KSUM)

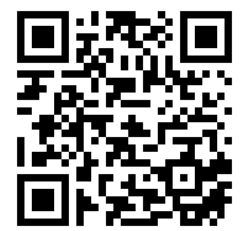

How to cite this article:

Jeon SK, Joo I, Kim SY, Jang JK, Park J, Park HS, et al. Quantitative ultrasound radiofrequency data analysis for the assessment of hepatic steatosis using the controlled attenuation parameter as a reference standard. Ultrasonography. 2021 Jan;40(1):136-146. 


\section{Introduction}

Hepatic steatosis is the key histologic feature of nonalcoholic fatty liver disease (NAFLD) and is associated with other chronic liver diseases such as alcoholic liver disease, chronic viral hepatitis, and drug-induced liver injury [1]. NAFLD is a growing public health problem worldwide. It can progress to nonalcoholic steatohepatitis, one of the leading indications for liver transplantation [2]. Hepatic steatosis accompanied by chronic viral hepatitis may increase the risk of developing cirrhosis and hepatocellular carcinoma [3]. However, hepatic steatosis can be improved by proper management $[4,5]$. Therefore, early detection and accurate staging of hepatic steatosis are important for timely management, treatment monitoring, and predicting the prognosis.

Although liver biopsy has been regarded as the gold standard for hepatic steatosis, it has drawbacks such as invasiveness, sampling errors, and inter-reader variability. Hence, a noninvasive diagnostic tool is required. B-mode ultrasonography (US) has been widely used as a primary imaging modality for hepatic steatosis. Conventionally, hepatic steatosis has been subjectively estimated using B-mode US, but it has limitations due to inter-observer variability and poor sensitivity for mild steatosis [6]. The hepatorenal index (HRI), defined as the ratio of liver to kidney echointensities measured on B-mode US, has been developed as a quantitative parameter for evaluating hepatic steatosis, but its use is limited in patients with renal disease or a heterogeneous distribution of steatosis [7]. Magnetic resonance (MR) techniques such as the MR spectroscopy (MRS) and MR imaging (MRI)-determined proton density fat fraction have been extensively validated as an appropriate reference standard $[8,9]$. However, both MRS and MRI are expensive and not routinely accessible. The controlled attenuation parameter (CAP), a measurement of ultrasonic attenuation vibration obtained in transient elastography (TE), has been recently suggested as an alternative [10]. Although CAP is less accurate than the MRS or MRI-determined proton density fat fraction for diagnosing hepatic steatosis [11], CAP has the advantage of being inexpensive and relatively widely available.

Recently, increasing attention has been paid to quantitative US radiofrequency (RF) data analysis as a promising tool for tissue characterization. As opposed to B-mode images, raw RF data contain frequency-dependent information of the US signal, which provides additional diagnostic value $[12,13]$, and it can be obtained by turning on a specific acquisition mode. Recent studies have demonstrated that some quantitative parameters from the RF data analysis reflecting the backscatter or attenuation of the US beam correlated well with hepatic steatosis grades [14-17]. Additionally, those parameters have been shown to be more accurate than qualitative visual scores on B-mode US to diagnose steatosis grades [15]. However, little is known about the diagnostic performance of $\mathrm{RF}$ data-driven parameters for hepatic steatosis and their potential confounding factors.

In this study, we aimed to investigate the diagnostic value of quantitative US parameters from RF data analysis for assessing hepatic steatosis, using CAP-based steatosis grades as the reference standard.

\section{Materials and Methods}

\section{Study Population}

This prospective study was approved by each participating center, and written informed consent was obtained from all participants. Our study population was a subgroup of a prospective multicenter study that primarily aimed to evaluate the performance of point shear-wave elastography (SWE) for hepatic fibrosis [18] (ClinicalTrials.gov identifier: NCT03047707). In the main study, participants were enrolled from May 2017 to April 2018 and underwent both B-mode US with point SWE and TE. Its inclusion criteria were (1) patients with chronic liver disease or liver cirrhosis, patients scheduled to undergo hepatectomy for liver disease or liver donation, or healthy volunteers; and (2) age $\geq 18$ years old. Patients were excluded if they had (1) obstructive cholestasis, (2) high serum aspartate aminotransferase and/or alanine aminotransferase levels ( $>5$ times the upper normal limit) within 3 months, (3) right heart failure or liver congestion, (4) previous liver surgery, or (5) infiltrative liver disease. Among these patients, those who had reliable CAP measurements within a 2-week interval from B-mode US were selected for this study. While four centers participated in the main study, the TE devices at only two centers provided CAP values in addition to liver stiffness measurements (LSMs). Therefore, our study participants were enrolled at either of those two centers (Seoul National University Hospital and Asan Medical Center, Seoul, Korea).

\section{Acquisition of B-Mode US and TE}

For each participant, B-mode US and TE were performed by different experienced operators who were blinded to the results of the other study. Before B-mode US or TE, participants were requested to fast for at least 4 hours.

\section{$B$-mode US imaging with RF data}

All B-mode US examinations were performed with a diagnostic US system (RS80A, Samsung Medison, Co. Ltd., Seoul, Korea) using a convex probe (CA1-7A). Using a predefined preset with S-Harmonic mode (pulse inversion+coded harmonic imaging), B-mode images were obtained during a breath-hold with a fixed setting of time-gain 
compensation, and their RF data were automatically recorded. The scan planes included a right intercostal plane near the level of the hepatic hilum and a right sagittal plane showing both the liver and right kidney. The right intercostal plane was for the RF data analysis, while the right sagittal plane was for the HRI measurement [19].

\section{TE with CAP}

Using TE (Fibroscan, Echosens, Paris, France), CAP (in $\mathrm{dB} / \mathrm{m}$ ), and LSM (in $\mathrm{kPa}$ ) were measured with an $\mathrm{M}$ probe according to the manufacturer's recommendations. For each participant, the median of 10 valid measurements was regarded as representative of CAP and $L S M$, respectively [20]. The values were considered to be reliable when 10 valid measurements were made with an interquartile range $(\mathrm{IQR})<40 \mathrm{~dB} / \mathrm{m}$ for CAP [21] and an IQR/median ratio $\leq 30 \%$ for LSM [22].

\section{Image Analysis}

Visual scoring, HRI measurements, and RF data analysis were separately performed by abdominal radiologists who were blinded to the other results. To minimize recall bias in radiologists who participated in more than one assessment listed above, the assessments for each patient were separated by at least 4 weeks.

\section{Visual scoring of hepatic steatosis on B-mode US}

During the B-mode US examination, the visual score of hepatic steatosis was recorded by the operator (I.J. or J.M.L. for the participants at Seoul National University Hospital and S.Y.K. or J.K.J. for the participants at Asan Medical Center). The visual score of hepatic steatosis was graded as follows: score 0, no steatosis; 1, mild steatosis; 2, moderate steatosis; and 3, severe steatosis) by referring to Hamaguchi's scoring system [23], which uses the following US features of hepatic steatosis: bright liver, increased hepatorenal echo contrast, deep attenuation, and vessel blurring.

\section{HRI measurements on B-mode US}

The HRI was calculated by analyzing B-mode US images on a picture archiving and communication system by an abdominal radiologist (S.K.J. for the participants at Seoul National University Hospital and S.Y.K. for the participants at Asan Medical Center). A circular region of interest (ROI) was drawn in the liver parenchyma, and another one was drawn in the renal parenchyma at the same depth as the $\mathrm{ROI}$ in the liver. The liver ROI should avoid focal lesions, large vessels, and bile ducts, and the kidney ROI should avoid focal lesions, collecting systems, and extra-renal tissues. The average echointensity of the liver ROI was divided by that of the kidney ROI to calculate the HRI [19]. The HRI measurement was defined as a failure when the liver ROI and the kidney ROI could not be placed at the same depth or when an ROI of at least $20 \mathrm{~mm}^{2}$ could not be drawn in the liver or the kidney.

\section{Measurement of quantitative US parameters from RF data analysis}

Two quantitative US parameters of the liver parenchyma, including the tissue scatter-distribution imaging parameter (TSI-p) and tissue attenuation imaging parameter (TAI-p), were derived from the RF data. TSI-p indicates the average Nakagami parameters of the ROI, reflecting the local concentration and arrangement of US scatterers [14]. TAI-p indicates the slope of the US center frequency downshift with a depth that can be used to estimate acoustic attenuation [24]. The theoretical background of these two parameters and details on how to create the parametric maps are given in the Supplementary Data 1.

By analyzing RF data using an in-house program developed in MATLAB R2015a (MathWorks, Inc., Natick, MA, USA), color-coded maps of the Nakagami parameters (TSI map) and center frequency (TAI map) were generated on a pixel-by-pixel basis (Fig. 1). With reference to the B-mode image, a rectangular ROI (about $2 \mathrm{~cm}$ in width $\times 4 \mathrm{~cm}$ in height) in the TSI map and an annulus-sector ROI (about $2 \mathrm{~cm}$ in inner arc length $\times 4 \mathrm{~cm}$ in side length) in the TAI map were positioned in the liver parenchyma, avoiding large vessels, focal lesions, and reverberation artifacts beneath the liver capsule (Fig. 1), to calculate TSI-p and TAI-p.

Measurements of TSI-p and TAI-p were performed in two separate sessions by one radiologist (S.K.J.) at least 2 weeks apart and in one session by another radiologist (J.P.) to evaluate intra-observer and inter-observer reliability, respectively. The results of the first session of the former radiologist were used as representative values of each participant for the other analysis in this study.

\section{Determination of Hepatic Steatosis Grades and Hepatic Fibrosis Grade}

CAP values were used to determine hepatic steatosis grades by applying the reference values suggested in a previous study [20]: $0-250 \mathrm{~dB} / \mathrm{m}$ for $\mathrm{SO}$ (no steatosis), $>250 \mathrm{~dB} / \mathrm{m}$ for $\geq S 1$ (mild steatosis), $>299 \mathrm{~dB} / \mathrm{m}$ for $\geq \mathrm{S} 2$ (moderate steatosis), and $>327 \mathrm{~dB} /$ $\mathrm{m}$ for $\mathrm{S3}$ (severe steatosis). The LSM values on TE were used to determine hepatic fibrosis grades by applying the cut-offs suggested in a previous study [25]: $0-7.1 \mathrm{kPa}$ for $\leq \mathrm{F} 1$ (no or mild fibrosis), $>7.1 \mathrm{kPa}$ for $\geq \mathrm{F} 2$ (significant fibrosis), $>9.5 \mathrm{kPa}$ for $\geq \mathrm{F3}$ (severe fibrosis), and $>12.5 \mathrm{kPa}$ for cirrhosis (F4).

\section{Statistical Analysis}

The visual scores, HRI, TSI-p, and TAI-p were correlated with CAPbased steatosis grades using Spearman correlation analysis. The 


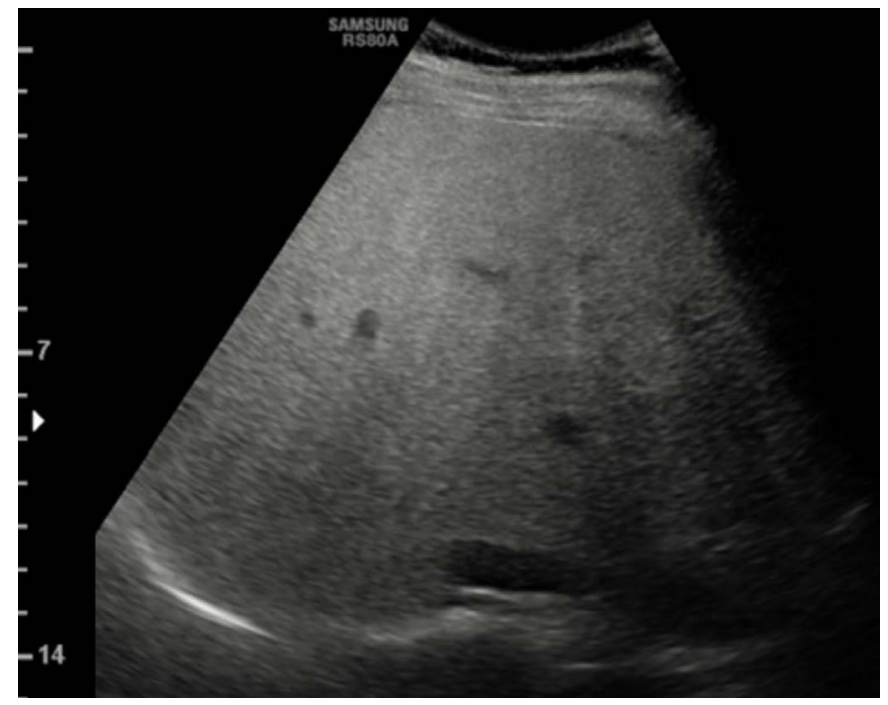

A

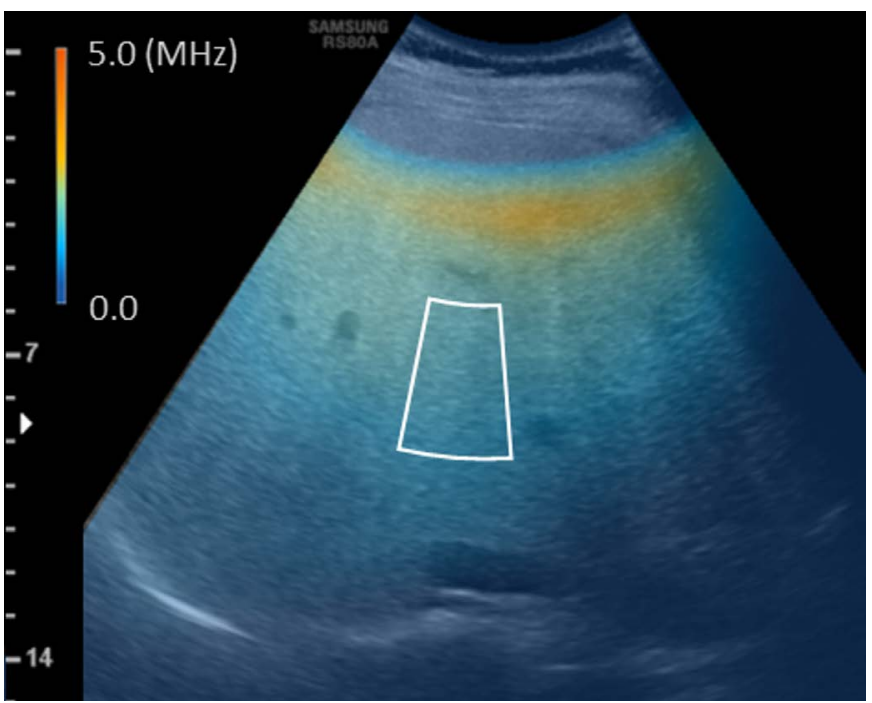

C

Spearman correlation coefficient $(\rho)$ was interpreted as follows: $|\rho|>0.5$, strong; $|\rho|=0.3-0.5$, moderate; and $|\rho|<0.3$, weak correlation [26]. As the Kolmogorov-Smirnov test revealed that the visual scores, HRI, and TAI-p were not normally distributed, the US parameters of different steatosis grades were compared with the Kruskal-Wallis test followed by the Dunn post-hoc test without the assumption of a normal distribution of data. In the Dunn posthoc test, a Bonferroni-adjusted P-value less than 0.017 (0.05/3) was considered to indicate statistical significance, as three pairwise comparisons between adjacent grades were made. The diagnostic performance of US parameters for detecting $\geq \mathrm{S} 1, \geq \mathrm{S} 2$, and $\mathrm{S} 3$ was assessed using nonparametric receiver operating characteristic (ROC) curve analyses. Pairwise comparisons of the areas under the ROC curve (AUCs) between parameters were performed using

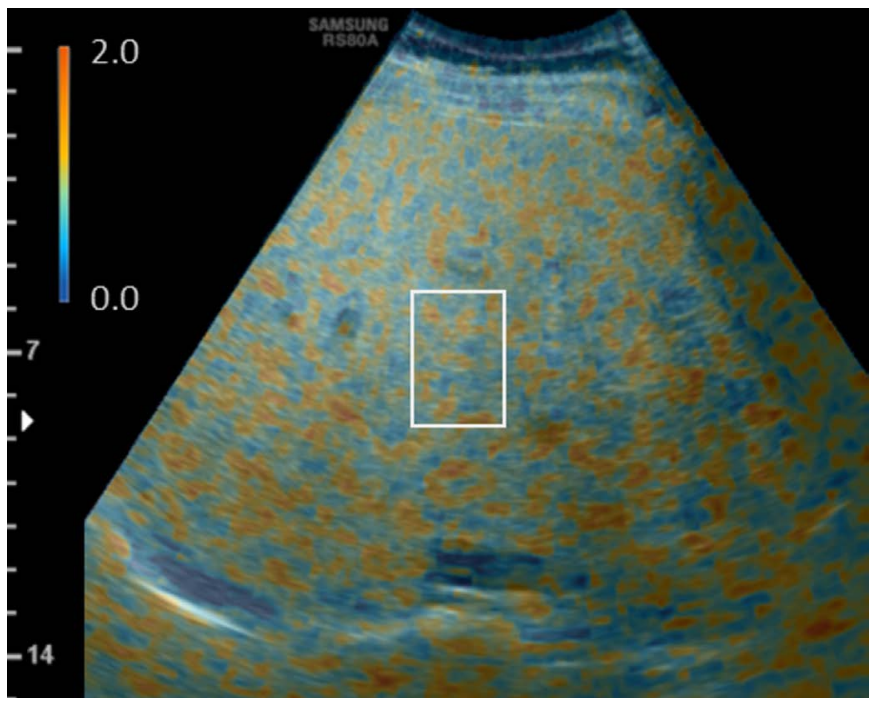

B

Fig. 1. Measurement of the quantitative ultrasound parameters of the liver parenchyma from radiofrequency data analysis.

From the simultaneously acquired radiofrequency data of a B-mode ultrasound image (A), a color-coded tissue scatter-distribution imaging (TSI) map reflecting the Nakagami parameters (B) and tissue attenuation imaging (TAI) map reflecting center frequency (V) were generated using an in-house program. By drawing regions of interests (ROIs) in the liver parenchyma in the maps of (B) and (C), respectively, the TSI parameter (the average of Nakagami parameters of the ROI) and TAI parameter (the slope of the ultrasound center frequency downshift with depth) were obtained.

the z-score, accounting for standard errors in the differences in correlated AUCs estimated by the Delong test. In this pairwise comparison analysis, a Bonferroni-adjusted P-value of less than $0.008(0.05 / 6)$ was considered to indicate statistical significance, as six pairwise comparisons were possible with the four different US parameters. The sensitivity and specificity for detecting $\geq S 1, \geq S 2$, and $\mathrm{S} 3$ were calculated based on the visual scores $(\geq 1, \geq 2$, and 3 , respectively), HRI (using cut-offs of $\geq 1.05, \geq 1.25$, and $\geq 1.65$, respectively [7]), TSI-p, and TAI-p (using cut-offs determined on the ROC curve analysis in this study as the points of maximizing the Youden index), respectively. Furthermore, to assess the potential effect of hepatic fibrosis on the diagnostic performance of TSI-p or TAI-p for hepatic steatosis, their AUCs were compared according to the presence of significant hepatic fibrosis using the method 
detailed by DeLong et al. [27]. Intra- and inter-observer reliability were assessed using intra-class correlation coefficients (ICCS) and interpreted as follows: $\geq 0.90$, excellent; $\geq 0.75$ to $<0.90$, good; $\geq 0.50$ to $<0.75$, moderate; and $<0.50$, poor reliability [28]. The coefficient of variation $(\mathrm{CV})$, the ratio of the standard deviation to the mean, was also calculated to provide an additional estimation of intra- and inter-observer reliability, with a small CV value indicating more reliable measurements [29]. Univariate and multivariate linear regression analyses were performed to evaluate significant determinants of TSI-p and TAI-p. Statistical analyses were performed using MedCalc 16.4.1 (MedCalc Software, Ostend, Belgium) and SPSS version 25.0 (IBM Corp., Armonk, NY, USA). A P-value of less than 0.05 was considered to indicate statistical significance, except for the aforementioned pairwise comparison tests.

\section{Results}

\section{Study Population}

Of the 249 participants who were initially enrolled, six participants with unreliable CAP measurements were excluded from the analysis. Therefore, a total of 243 participants (98 at Seoul National University Hospital and 145 at Asan Medical Center; 171 men; age, mean \pm standard deviation [SD], 55 \pm 13 years old; and body mass index [BMI], mean $\pm S D, 25 \pm 4 \mathrm{~kg} / \mathrm{m}^{2}$ ) were finally included. Patients' characteristics are summarized in Table 1. The majority had chronic liver diseases $(82.7 \%, 201$ of 243), with the most common cause being chronic hepatitis B $(47.7 \%, 116$ of 243). Based on the CAP values, 152, 54, 14, and 23 patients were categorized as having SO, $\mathrm{S} 1, \mathrm{~S} 2$, and $\mathrm{S} 3$. The number of patients with $\geq \mathrm{S} 1, \geq \mathrm{S} 2$, and $\mathrm{S} 3$ were $91(37.4 \%), 37(15.2 \%)$, and $23(9.5 \%)$, respectively. None of the patients showed unreliable LSM results, and 98 patients (40.3\%) were categorized as having $\geq F 2$ based on TE results. Among the final study population, the HRI measurement failed in five patients $(\mathrm{SO}, \mathrm{n}=3$ and $\mathrm{S1}, \mathrm{n}=2)$. Therefore, the HRI-related analysis was performed in 238 patients.

\section{Correlation of US Parameters with Hepatic Steatosis Grades} All the assessed US parameters showed significant correlations with hepatic steatosis grades (Table 2). The visual score and HRI showed moderate and weak positive correlations with steatosis grades, respectively $(\rho=0.352$ and $\rho=0.264$, respectively; $P<0.001$ for both), while TSI-p and TAI-p showed strong positive and negative correlations, respectively $(\rho=0.593$ and $\rho=-0.617$, respectively; $\mathrm{P}<0.001$ for both).

\section{Comparison of US Parameters According to Hepatic Steatosis Grades}

The US parameters according to hepatic steatosis grades are shown in Table 2 and Fig. 2. All the assessed parameters showed significant differences according to steatosis grades $(P<0.001$ for all) (Table 2$)$. When comparing between $S 0$ and S1, both TSI-p and TAI-p showed significant differences between groups $(P<0.001$ for both), while neither the visual scores nor HRI did so $(P=0.594$ and $P>0.99$, respectively). On the contrary, none of the parameters showed statistically significant differences between $S 1$ and $S 2(P \geq 0.114)$ or between S2 and S3 ( $P>0.99)$.

\section{Diagnostic Performance of US Parameters for Hepatic Steatosis Grades}

For the prediction of $\geq S 1, \geq S 2$, and $S 3$, TSI-p showed AUCs of 0.827 , 0.914 , and 0.917 , respectively. TAI-p showed AUCs of $0.844,0.914$, and 0.909, respectively (Table 3). Pairwise comparison analyses of the AUCs between US parameters were performed in participants

Table 1. Patients' characteristics

\begin{tabular}{|c|c|}
\hline Characteristic & Value $(n=243)$ \\
\hline Age (y) & $55 \pm 13(18-83)$ \\
\hline Sex (male: female) & $171: 72$ \\
\hline Body mass index $\left(\mathrm{kg} / \mathrm{m}^{2}\right)$ & $25 \pm 4(17-31)$ \\
\hline Skin-liver capsule distance (mm) & $18 \pm 4(10-36)$ \\
\hline \multicolumn{2}{|l|}{ Etiology of chronic liver disease } \\
\hline Chronic hepatitis B & $116(47.7)$ \\
\hline Chronic hepatitis C & $41(16.9)$ \\
\hline Alcoholic liver disease & $10(4.1)$ \\
\hline Unknown or other causes & $34(14.0)$ \\
\hline No underlying liver disease & $42(17.3)$ \\
\hline Aspartate aminotransferase (IU/L) & $30 \pm 17(10-161)$ \\
\hline Alanine aminotransferase (IU/L) & $30 \pm 18(5-117)$ \\
\hline \multicolumn{2}{|l|}{ Hepatic fibrosis grades } \\
\hline F0 or F1 ( $\leq 7.1 \mathrm{kPa}$ on TE) & $145(59.7)$ \\
\hline $\mathrm{F} 2$ (>7.1 to $\leq 9.5 \mathrm{kPa}$ on TE) & $31(12.8)$ \\
\hline F3 (>9.5 to $\leq 12.5 \mathrm{kPa}$ on TE) & $25(10.3)$ \\
\hline F4 (>12.5 kPa on TE) & $42(17.3)$ \\
\hline \multicolumn{2}{|l|}{ Hepatic steatosis grades } \\
\hline $\mathrm{SO}(\leq 250 \mathrm{~dB} / \mathrm{m}$ on $\mathrm{CAP})$ & $152(62.6)$ \\
\hline $\mathrm{S} 1$ (>250 to $\leq 299 \mathrm{~dB} / \mathrm{m}$ on CAP) & $54(22.2)$ \\
\hline S2 (>299 to $\leq 327 \mathrm{~dB} / \mathrm{m}$ on CAP) & $14(5.8)$ \\
\hline $\mathrm{S} 3$ (>327 dB/m on CAP) & $23(9.5)$ \\
\hline
\end{tabular}


Table 2. Ultrasound parameters according to hepatic steatosis grades

\begin{tabular}{|c|c|c|c|c|c|c|c|c|c|c|}
\hline \multirow{3}{*}{$\begin{array}{l}\text { Ultrasound } \\
\text { parameter }\end{array}$} & \multicolumn{5}{|c|}{ Hepatic steatosis grade } & \multirow{2}{*}{\multicolumn{3}{|c|}{$\begin{array}{c}\text { P-value } \\
\text { Dunn's post hoc test }\end{array}$}} & \multicolumn{2}{|c|}{$\begin{array}{c}\text { Spearman correlation } \\
\text { analysis }\end{array}$} \\
\hline & \multirow{2}{*}{ So $(n=152)$} & \multirow{2}{*}{$S 1(n=54)$} & \multirow{2}{*}{$S 2(n=14)$} & \multirow{2}{*}{$S 3(n=23)$} & \multirow{2}{*}{$\begin{array}{l}\text { Kruskal- } \\
\text { Wallis test }\end{array}$} & & & & \multirow{2}{*}{$\rho(95 \% \mathrm{Cl})$} & \multirow{2}{*}{ P-value } \\
\hline & & & & & & S0 vs. S1 & S1 vs. S2 & S2 vs. S3 & & \\
\hline $\begin{array}{l}\text { Visual score } \\
(0-3)\end{array}$ & $0.45 \pm 0.04$ & $0.65 \pm 0.20$ & $1.21 \pm 0.24$ & $1.48 \pm 0.18$ & $<0.001^{\mathrm{a})}$ & 0.594 & 0.131 & $>0.999$ & $\begin{array}{c}0.352 \\
(0.236 \text { to } 0.458)\end{array}$ & $<0.001^{\text {a) }}$ \\
\hline$\left.H R\right|^{b)}$ & $1.28 \pm 0.04$ & $1.42 \pm 0.09$ & $1.96 \pm 0.29$ & $1.74 \pm 0.11$ & $<0.001^{a)}$ & $>0.999$ & 0.177 & $>0.999$ & $\begin{array}{c}0.264 \\
(0.139 \text { to } 0.381)\end{array}$ & $<0.001^{\mathrm{a})}$ \\
\hline TSI-p & $0.76 \pm 0.01$ & $0.88 \pm 0.02$ & $1.00 \pm 0.03$ & $1.04 \pm 0.02$ & $<0.001^{\mathrm{a})}$ & $<0.001^{\mathrm{a})}$ & 0.114 & $>0.999$ & $\begin{array}{c}0.593 \\
(0.505 \text { to } 0.669)\end{array}$ & $<0.001^{\text {a) }}$ \\
\hline $\begin{array}{l}\text { TAl-p } \\
(\mathrm{MHz} / \mathrm{cm})\end{array}$ & $-0.06 \pm 0.01$ & $-0.09 \pm 0.01$ & $-0.13 \pm 0.01$ & $-0.13 \pm 0.01$ & $<0.001^{\text {a) }}$ & $<0.001^{\mathrm{a})}$ & 0.139 & $>0.999$ & $\begin{array}{c}-0.617 \\
(-0.690 \text { to }-0.533)\end{array}$ & $<0.001^{\text {a) }}$ \\
\hline
\end{tabular}

Values are presented as mean \pm standard deviation unless otherwise specified.

$\mathrm{Cl}$, confidence interval; $\mathrm{HRI}$, hepatorenal index; TSI-p, tissue scatter-distribution imaging parameter; TAI-p, tissue attenuation imaging parameter.

${ }^{\text {a) }}$-values that indicate statistical significance $(<0.05$, except for post hoc test: $<0.017[0.05 / 3$, according to the Bonferroni correction]). b) HRI analysis was performed in 238 patients ( $S 0, n=149 ; S 1, n=52 ; S 2, n=14 ; S 3, n=23$ ) as the measurement failed in 5 patients. Visual scores of hepatic steatosis grades were $0 / 1 / 2 / 3$ for no/mild/moderate/ severe steatosis.

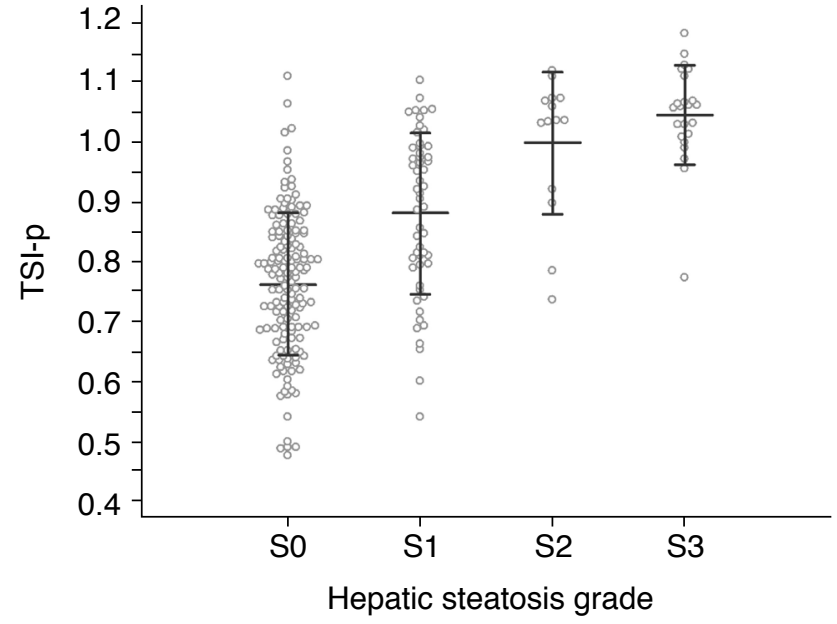

A

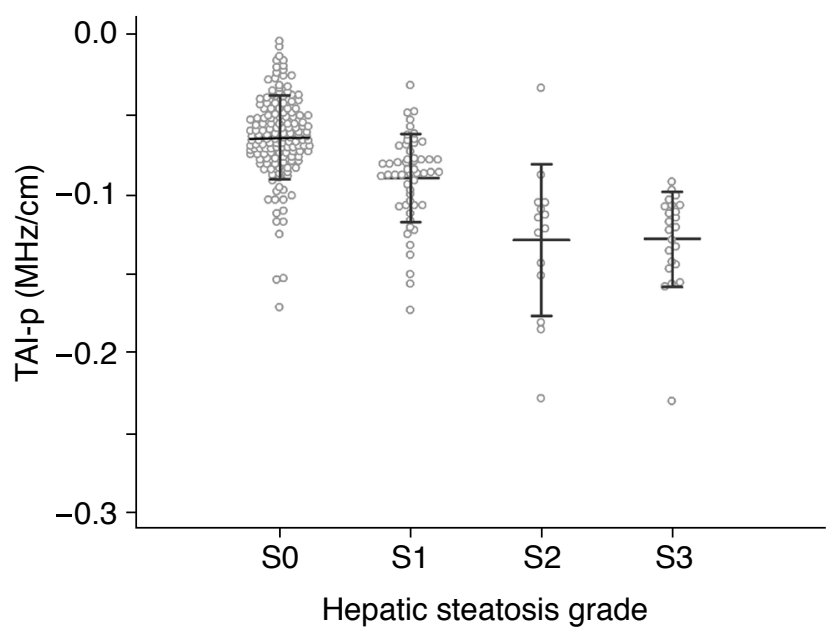

B

Fig. 2. Scatter dot plots of the tissue scatter-distribution imaging parameter (TSI-p) (A) and tissue attenuation imaging parameter (TAI-p) (B) according to the hepatic steatosis grade. Error bars represent the standard deviation from the mean.

with available values. The visual scores, HRI, TSI- $p$, and TAI- $p(n=238)$, are described in Supplementary Table 1. Both TSI-p and TAI-p showed significantly higher AUCs than either the visual scores or HRI for diagnosing $\geq S 1$ or $\geq S 2$ ( $P \leq 0.003$ for all). For the diagnosis of S3, both TSI-p and TAI-p also showed higher AUCS than the visual scores or $H R I$, with statistical significance $(P \leq 0.029$ for all). The sensitivity and specificity for $\geq \mathrm{S} 1, \geq \mathrm{S} 2$, and $\mathrm{S} 3$ using the cut-off values for each US parameter are described in Table 3. For detecting $\geq S 1$, a TSI-p $>0.910$ resulted in a sensitivity of $65.9 \%$ (60 of 91 ) and a specificity of $92.8 \%$ (141 of 152), while a TAI-p $\leq-0.078 \mathrm{MHz} /$ $\mathrm{cm}$ resulted in a sensitivity of $83.5 \%$ (76 of 91 ) and a specificity of $77.6 \%$ (118 of 152$)$.

\section{Clinical and Laboratory Determinants of TSI-p and TAI-p}

In the univariate linear regression analysis, BMI, TE-based fibrosis grade, and CAP-based steatosis grade were significant factors affecting TSI-p. Additionally, BMI, skin-liver capsule distance measured on B-mode US, alanine aminotransferase levels, and the CAP-based steatosis grade were significant factors affecting TAI- $p$ (Table 4). In the multivariate analysis, the fibrosis grade and steatosis grade were independent determinants for TSI-p, showing negative and positive relationships $(P=0.034$ and $P<0.001)$, respectively. The steatosis grade was an independent determinant for TAI-p, with a negative relationship $(\mathrm{P}<0.001)$ (Table 4).

For $\geq S 1, \geq S 2$, and $S 3$, the AUCS of TSI-p and TAI-p in patients 
without significant fibrosis were higher than those in patients with significant fibrosis (Supplementary Table 2). However, a statistically significant difference between subgroups was found only in TSI-p for detecting $\geq S 1$ ( $A U C, 0.897$ vs. $0.705 ; P=0.003$ ).
Intra- and Inter-observer Reliability of TSI-p and TAI-p

On the given parametric maps, the intra-observer reliability of TSI-p and TAI-p measurements of the liver parenchyma was excellent, with ICCs of 0.989 and 0.968 and CVs of $2.9 \%$ and $13.0 \%$, respectively. The inter-observer reliability of TSI-p and TAI-p was also excellent, with ICCS of 0.944 and 0.945 and CVs of $11.4 \%$ and $23.6 \%$,

Table 3. Diagnostic performance of US parameters in the prediction of hepatic steatosis grades

\begin{tabular}{|c|c|c|c|c|c|}
\hline US parameter & Hepatic steatosis grade & AUC (95\% Cl) & Cut-off & Sensitivity, n (\%) & Specificity, n (\%) \\
\hline \multirow[t]{3}{*}{ Visual score (0-3) } & $\geq S 1$ & 0.659 (0.596 to 0.719$)$ & $\geq$ Score 1 (mild) & $58 / 91(63.7)$ & $86 / 152(56.6)$ \\
\hline & $\geq S 2$ & 0.778 (0.721 to 0.829$)$ & $\geq$ Score 2 (moderate) & $19 / 37(51.4)$ & 198/206 (96.1) \\
\hline & S3 & 0.794 (0.737 to 0.843 ) & Score 3 (severe) & $2 / 23(8.7)$ & $220 / 220(100)$ \\
\hline \multirow[t]{3}{*}{$\left.H R\right|^{a)}$} & $\geq S 1$ & 0.629 (0.564 to 0.691$)$ & $\geq 1.05$ & $72 / 89(80.9)$ & $39 / 149(26.2)$ \\
\hline & $\geq S 2$ & 0.751 (0.692 to 0.805$)$ & $\geq 1.25$ & $27 / 37(73.0)$ & $117 / 127(92.1)$ \\
\hline & S3 & 0.759 (0.700 to 0.812$)$ & $\geq 1.65$ & $11 / 23(47.8)$ & $177 / 215(82.3)$ \\
\hline \multirow[t]{3}{*}{ TSI-p } & $\geq S 1$ & 0.827 (0.773 to 0.872 ) & $>0.910$ & $60 / 91(65.9)$ & $141 / 152(92.8)$ \\
\hline & $\geq S 2$ & 0.914 (0.871 to 0.946$)$ & $>0.952$ & $32 / 37(86.5)$ & 179/206 (86.9) \\
\hline & S3 & 0.917 (0.875 to 0.948$)$ & $>0.952$ & $22 / 23(95.7)$ & $183 / 220(83.2)$ \\
\hline \multirow[t]{3}{*}{ TAI-p (MHz/cm) } & $\geq S 1$ & 0.844 (0.793 to 0.888 ) & $\leq-0.078$ & $76 / 91(83.5)$ & $118 / 152(77.6)$ \\
\hline & $\geq S 2$ & 0.914 (0.872 to 0.946$)$ & $\leq-0.093$ & $34 / 37(91.9)$ & $173 / 206(84.0)$ \\
\hline & S3 & 0.909 (0.866 to 0.942$)$ & $\leq-0.093$ & $22 / 23(95.7)$ & $175 / 220(79.6)$ \\
\hline
\end{tabular}

US, ultrasonography; AUC, area under the receiver operating characteristic curve; $\mathrm{Cl}$, confidence interval; HRI, hepatorenal index; TSI-p, tissue scatter-distribution imaging parameter; TAI-p, tissue attenuation imaging parameter.

${ }^{a)} H R I$ analysis was performed in 238 patients $(S 0, n=149 ; S 1, n=52 ; S 2, n=14 ; S 3, n=23)$ as the measurement failed in 5 patients.

Table 4. Univariate and multivariate linear regression analysis for identifying determinants of ultrasound radiofrequency data-driven parameters of the liver parenchyma

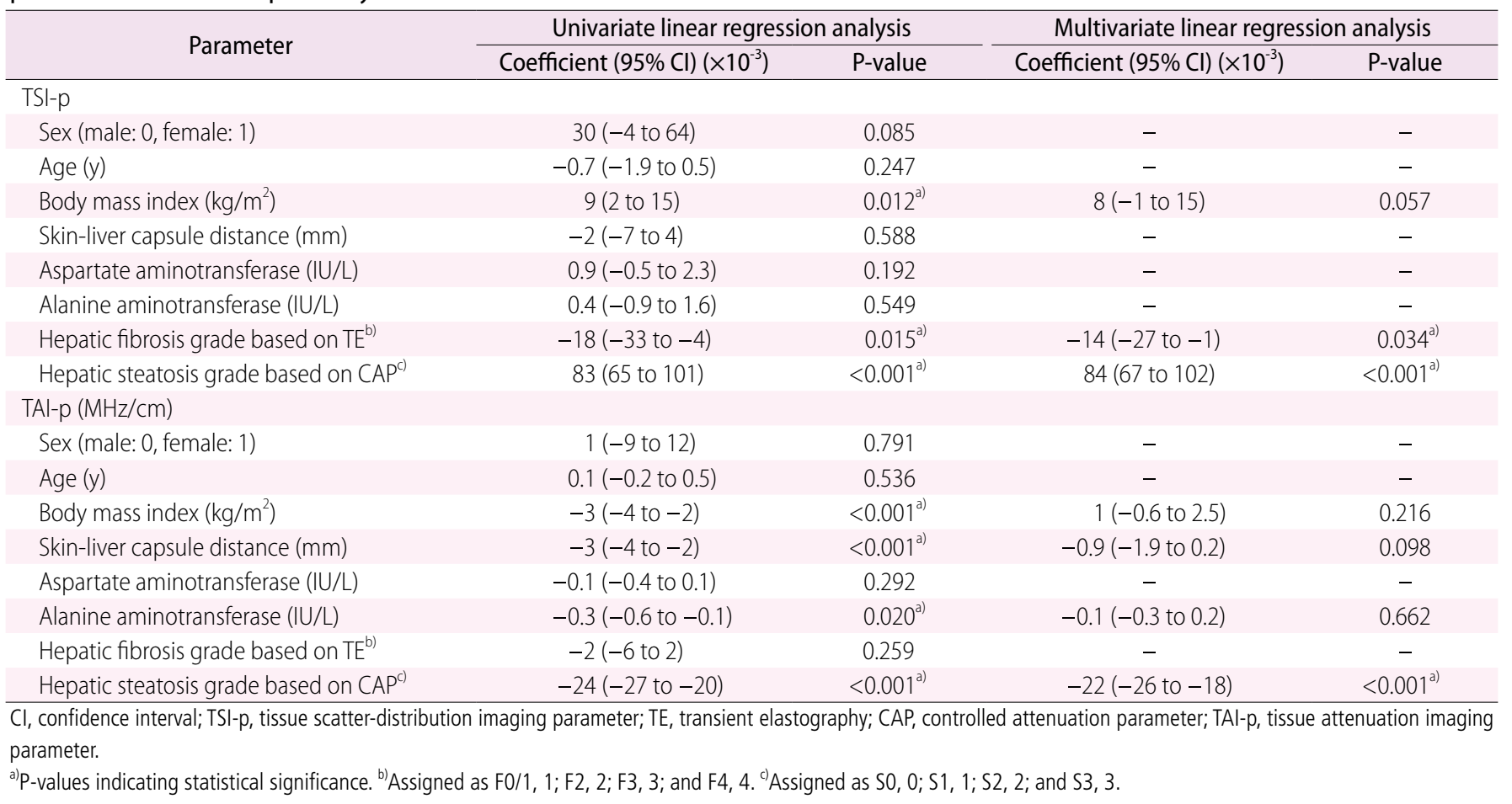


respectively (Supplementary Table 3).

\section{Discussion}

Our study of patients with chronic liver diseases showed that TSI-p and TAI-p of the liver parenchyma, which are quantitative US parameters from RF data analysis, were well correlated with CAPbased hepatic steatosis grades and that each of those parameters performed well in diagnosing $\geq$ mild (S1), $\geq$ moderate (S2), and severe (S3) steatosis. Moreover, both TSI-p and TAI-p showed significantly higher AUCs than the visual scores or HRI for predicting $\geq S 1$ or $\geq S 2$. The multivariate linear regression analysis revealed that the hepatic steatosis grade was an independent determinant of both TSI-p and TAI- $p$ and that the hepatic fibrosis grade was also an independent determinant of TSI-p. The measurements of TSI-p and TAI-p showed excellent intra- and inter-observer reliability. These results support the clinical applicability of TSI-p and TAI-p for assessing hepatic steatosis.

In our study, TSI-p and TAI-p showed significant positive and negative correlations with steatosis grade, respectively ( $\rho$ $=0.593$ and $\rho=-0.617 ; P<0.001$ for both). These findings can be explained by the interaction of the US beam with liver tissue. If the concentration of fat droplets (the acoustic scatterers) increases in a homogeneous medium such as the liver parenchyma, the US backscattered statistics shift from a pre-Rayleigh to a Rayleigh distribution, which increases TSI-p [14]. Moreover, when the amount of fat droplets increases in the liver, US beam attenuation along the acoustic path becomes more severe, especially for the high-frequency beam components [24]. This supports our findings of a negative correlation between the steatosis grade and TAI-p. Given their strong relationships with the steatosis grade as shown in our study, TSI-p and TAI-p are expected to be useful in diagnosing hepatic steatosis. TSI-p and TAI-p showed good diagnostic performance in our study, with AUCs of 0.827 and 0.844 for $\geq S 1 ; 0.914$ and 0.914 for $\geq S 2 ; 0.917$ and 0.909 for $S 3$, respectively. These results agree with those of previous studies of US backscattering or attenuationrelated parameters in NAFLD or other chronic liver diseases (AUCS of $0.84-0.90$ for $\geq S 1 ; 0.89-0.95$ for $\geq S 2$; and $0.93-0.96$ for $\mathrm{S} 3$ ) $[30,31]$.

Early detection of mild hepatic steatosis has clinical implications as it enables timely lifestyle interventions that may prevent or delay the further progression of steatosis and associated comorbidities [32]. In our study, both TSI-p and TAI-p showed significant differences between $\mathrm{SO}$ and $\mathrm{S} 1$, while visual scores and HRI did not. Moreover, when applying the cut-offs determined for the ROC curve analysis, TSI-p and TAI-p provided balanced sensitivity and specificity in each (sensitivity, $65.9 \%$ and $83.5 \%$, respectively; specificity,
$92.8 \%$ and $77.6 \%$, respectively). Our results suggest the potential application of RF data-driven parameters as a screening method for hepatic steatosis. However, the cut-off values need to be further validated. Of note, the diagnostic performance of visual scores and HRI for hepatic steatosis in our study was somewhat poorer than has been reported in previous studies $[33,34]$. This difference could be associated with the characteristics of the study population, as the majority of our study population had chronic liver disease with or without hepatic steatosis, while previous studies $[33,34]$ focused on NAFLD patients.

According to the multivariate linear regression analysis in our study, the hepatic steatosis grade was an independent determinant of both TSI-p and TAI- $p$, with positive and negative relationships $(P=0.034$ and $P<0.001)$, respectively, and the hepatic fibrosis grade was an independent determinant of TSI- $p$, with a negative relationship $(P<0.001)$. However, the hepatic fibrosis grade did not show a significant relationship with TAI-p, even in the univariate analysis. Potential explanations for the relationships between steatosis and TSI-p or TAI-p are discussed above. The inverse relationship of hepatic fibrosis with TSI-p in our study is concordant with previous studies using Nakagami imaging $[35,36]$. This can be explained as follows: backscattered US signals from normal liver tissues mainly consist of randomly distributed scatterers, conforming to a near-Rayleigh distribution (pre-Rayleigh due to the vessel walls). Contrastingly, those from a cirrhotic liver with more resolvable scatterers (fibrotic structures and nodules) exhibit more of a pre-Rayleigh distribution, resulting in a decrease in the Nakagami parameter, the pixel-wise value of TSI-p [36,37]. Moreover, the diagnostic performance of TSI-p for $\geq S 1$ was significantly lower in patients with significant hepatic fibrosis than in those without significant fibrosis in our study. However, conflicting results have been reported on the effect of hepatic fibrosis on US attenuation. A previous study [38] revealed that hepatic fibrosis worsened US attenuation, while other studies $[30,39]$ have reported that hepatic fibrosis did not have a significant effect on US attenuation, similar to our study. Our results suggest that TSI-p is more strongly affected by hepatic fibrosis than TAI-p, and it is necessary to consider the effects of both steatosis and fibrosis when interpreting TSI-p. Given that SWE, an extensively validated technique for hepatic fibrosis [40], is now available on many diagnostic US machines, the addition of SWE data could be useful for accurately diagnosing hepatic steatosis using TSI-p.

There are several limitations to our study. Firstly, our study population was heterogeneous and had various chronic liver diseases. As steatosis patterns and other parenchymal changes may vary depending on the etiology of liver disease, the performance of US parameters need to be evaluated according to the etiology. 
Secondly, in our study, CAP-based steatosis grades were used as the reference standard. Although CAP is a well-validated tool for hepatic fat quantification [10], its values can be influenced by several covariates such as BMI and diabetes [40] and the reported cut-offs for steatosis grades vary across studies. To determine the associations of RF data-driven parameters with each histologic feature (e.g., steatosis, fibrosis, and inflammation, etc.), future studies with histologic results would be required. Thirdly, as all US examinations with RF data acquisition were performed using one US machine with a predefined preset, the reproducibility of quantitative RF data-driven parameters and the optimal scan settings should be further investigated.

In conclusion, TSI-p and TAI-p derived from US RF data may be useful in the detection and severity assessment of hepatic steatosis, which could be superior to B-mode US parameters. These results support the possibility that multi-parametric US examinations, including B-mode US, RF data analysis, and elastography may be a promising approach for a comprehensive assessment of liver pathologies, including steatosis as well as fibrosis, although further validation is required.

ORCID: Sun Kyung Jeon: https://orcid.org/0000-0002-8991-3986; ljin Joo: https:// orcid.org/0000-0002-1341-4072; So Yeon Kim: https://orcid.org/0000-0001-68538577; Jong Keon Jang: https://orcid.org/0000-0002-2938-6635; Juil Park: https:// orcid.org/0000-0002-5265-8723; Hee Sun Park: https://orcid.org/0000-0003-0869269X; Eun Sun Lee: https://orcid.org/0000-0003-0780-7985; Jeong Min Lee: https:// orcid.org/0000-0003-0561-8777

\section{Author Contributions}

Conceptualization: Joo I, Kim SY, Park HS, Lee ES, Lee JM. Data acquisition: Joo I, Kim SY, Jang JK, Lee JM. Data analysis or interpretation: Jeon SK, Joo I, Park J, Kim SY, Jang JK, Lee JM. Drafting of the manuscript: Jeon SK, Joo I. Critical revision of the manuscript: Kim SY, Lee JM. Approval of the final version of the manuscript: all authors.

\section{Conflict of Interest}

This study was supported by a research grant from Samsung Medison Co., Ltd. S.Y.K. received grants from Samsung Medison for this study, as well as personal fees and financial support from Samsung Medison and from Bayer Healthcare outside the study. J.K.J. received grants from Canon Medical Systems outside the submitted work. H.S.P. and E.S.L. received grants from Samsung Medison outside the submitted work. J.M.L. received grants from Samsung Medison for this study; personal fees and non-financial support from Siemens Healthcare, grants from RF MEDICAL, grants and personal fees from Bayer Healthcare, grants and personal fees from Guerbet, grants from Samsung Medison, grants from Philips Healthcare, grants from GE Healthcare, and grants from Canon Medical, outside the submitted work. All remaining authors have declared no conflicts of interest.

\section{Supplementary Material}

Supplementary Table 1. Comparison of diagnostic performance of ultrasound parameters for the assessment of hepatic steatosis grade (https://doi.org/10.14366/usg.20042).

Supplementary Table 2. TSI-p and TAI-p for the assessment of hepatic steatosis grades according to accompanying significant hepatic fibrosis (https://doi.org/10.14366/usg.20042).

Supplementary Table 3. Intra-observer and inter-observer reliability of TSI-p and TAI-p measurements (https://doi.org/10.14366/ usg.20042).

Supplementary Data 1. Quantitative ultrasound parameters from radiofrequency data analysis: theoretical background and how to create parametric maps (https://doi.org/10.14366/usg.20042).

\section{References}

1. Ma X, Holalkere NS, Kambadakone RA, Mino-Kenudson M, Hahn PF, Sahani DV. Imaging-based quantification of hepatic fat: methods and clinical applications. Radiographics 2009;29:1253-1277.

2. Wong RJ, Aguilar M, Cheung R, Perumpail RB, Harrison SA, Younossi $Z M$, et al. Nonalcoholic steatohepatitis is the second leading etiology of liver disease among adults awaiting liver transplantation in the United States. Gastroenterology 2015;148:547-555.

3. Veldt BJ, Chen W, Heathcote EJ, Wedemeyer H, Reichen J, Hofmann WP, et al. Increased risk of hepatocellular carcinoma among patients with hepatitis $\mathrm{C}$ cirrhosis and diabetes mellitus. Hepatology 2008:47:1856-1862.

4. Patton HM, Patel K, Behling C, Bylund D, Blatt LM, Vallee M, et al. The impact of steatosis on disease progression and early and sustained treatment response in chronic hepatitis C patients. J Hepatol 2004;40:484-490.

5. Friedman SL, Neuschwander-Tetri BA, Rinella M, Sanyal AJ. Mechanisms of NAFLD development and therapeutic strategies. Nat Med 2018;24:908-922.

6. Dasarathy S, Dasarathy J, Khiyami A, Joseph R, Lopez R, McCullough AJ. Validity of real time ultrasound in the diagnosis of hepatic steatosis: a prospective study. J Hepatol 2009;51:1061-1067.

7. Webb M, Yeshua H, Zelber-Sagi S, Santo E, Brazowski E, Halpern $Z$, et al. Diagnostic value of a computerized hepatorenal index for 
sonographic quantification of liver steatosis. AJR Am J Roentgenol 2009;192:909-914.

8. Zheng D, Guo Z, Schroder PM, Zheng Z, Lu Y, Gu J, et al. Accuracy of MR imaging and MR spectroscopy for detection and quantification of hepatic steatosis in living liver donors: a meta-analysis. Radiology 2017;282:92-102.

9. Dulai PS, Sirlin CB, Loomba R. MRI and MRE for non-invasive quantitative assessment of hepatic steatosis and fibrosis in NAFLD and NASH: clinical trials to clinical practice. J Hepatol 2016;65:1006-1016.

10. Eddowes PJ, Sasso M, Allison M, Tsochatzis E, Anstee QM, Sheridan $D$, et al. Accuracy of FibroScan controlled attenuation parameter and liver stiffness measurement in assessing steatosis and fibrosis in patients with nonalcoholic fatty liver disease. Gastroenterology 2019;156:1717-1730.

11. Park CC, Nguyen P, Hernandez C, Bettencourt R, Ramirez K, Fortney $L$, et al. Magnetic resonance elastography vs transient elastography in detection of fibrosis and noninvasive measurement of steatosis in patients with biopsy-proven nonalcoholic fatty liver disease. Gastroenterology 2017;152:598-607.

12. Nam K, Rosado-Mendez IM, Wirtzfeld LA, Ghoshal G, Pawlicki $A D$, Madsen EL, et al. Comparison of ultrasound attenuation and backscatter estimates in layered tissue-mimicking phantoms among three clinical scanners. Ultrason Imaging 2012;34:209-221.

13. Mamou J, Oelze ML. Quantitative ultrasound in soft tissues. New York: Springer, 2013.

14. Wan YL, Tai DI, Ma HY, Chiang BH, Chen CK, Tsui PH. Effects of fatty infiltration in human livers on the backscattered statistics of ultrasound imaging. Proc Inst Mech Eng H 2015;229:419-428.

15. Paige JS, Bernstein GS, Heba E, Costa EA, Fereirra M, Wolfson $T$, et al. A pilot comparative study of quantitative ultrasound, conventional ultrasound, and MRI for predicting histologydetermined steatosis grade in adult nonalcoholic fatty liver disease. AJR Am J Roentgenol 2017;208:W168-W177.

16. Yoo J, Lee JM, Joo I, Lee DH, Yoon JH, Kang HJ, et al. Reproducibility of ultrasound attenuation imaging for the noninvasive evaluation of hepatic steatosis. Ultrasonography 2020;39:121-129.

17. Lee DH, Lee JY, Park MS, Han JK. Non-invasive monitoring of hepatic steatosis via acoustic structure quantification of ultrasonography with MR spectroscopy as the reference standard. Ultrasonography 2020;39:70-78.

18. Joo I, Kim SY, Park HS, Lee ES, Kang HJ, Lee JM. Validation of a new point shear-wave elastography method for noninvasive assessment of liver fibrosis: a prospective multicenter study. Korean J Radiol 2019;20:1527-1535.

19. Shiralkar K, Johnson S, Bluth El, Marshall RH, Dornelles A, Gulotta PM. Improved method for calculating hepatic steatosis using the hepatorenal index. J Ultrasound Med 2015;34:1051-1059.

20. Chon YE, Jung KS, Kim SU, Park JY, Park YN, Kim DY, et al.
Controlled attenuation parameter (CAP) for detection of hepatic steatosis in patients with chronic liver diseases: a prospective study of a native Korean population. Liver Int 2014;34:102-109.

21. Wong VW, Petta S, Hiriart JB, Camma C, Wong GL, Marra F, et al. Validity criteria for the diagnosis of fatty liver by $\mathrm{M}$ probe-based controlled attenuation parameter. J Hepatol 2017;67:577-584.

22. Castera $L$, Forns $X$, Alberti A. Non-invasive evaluation of liver fibrosis using transient elastography. J Hepatol 2008;48:835-847.

23. Hamaguchi M, Kojima T, Itoh Y, Harano Y, Fujii K, Nakajima T, et al. The severity of ultrasonographic findings in nonalcoholic fatty liver disease reflects the metabolic syndrome and visceral fat accumulation. Am J Gastroenterol 2007;102:2708-2715.

24. Kim H, Varghese T. Attenuation estimation using spectral cross-correlation. IEEE Trans Ultrason Ferroelectr Freq Control 2007;54:510-519.

25. Castera L, Vergniol J, Foucher J, Le Bail B, Chanteloup E, Haaser M, et al. Prospective comparison of transient elastography, Fibrotest, APRI, and liver biopsy for the assessment of fibrosis in chronic hepatitis C. Gastroenterology 2005;128:343-350.

26. Kobus T, van der Laak JA, Maas MC, Hambrock T, Bruggink CC, Hulsbergen-van de Kaa CA, et al. Contribution of histopathologic tissue composition to quantitative MR spectroscopy and diffusionweighted imaging of the prostate. Radiology 2016;278:801-811.

27. DeLong ER, DeLong DM, Clarke-Pearson DL. Comparing the areas under two or more correlated receiver operating characteristic curves: a nonparametric approach. Biometrics 1988;44:837-845.

28. Koo TK, Li MY. A guideline of selecting and reporting intraclass correlation coefficients for reliability research. J Chiropr Med 2016;15:155-163.

29. Bland JM, Altman DG. Statistical methods for assessing agreement between two methods of clinical measurement. Lancet 1986;1:307310.

30. Bae JS, Lee DH, Lee JY, Kim H, Yu SJ, Lee JH, et al. Assessment of hepatic steatosis by using attenuation imaging: a quantitative, easy-to-perform ultrasound technique. Eur Radiol 2019;29:64996507.

31. Fujiwara $Y$, Kuroda H, Abe T, Ishida K, Oguri T, Noguchi S, et al. The B-mode image-guided ultrasound attenuation parameter accurately detects hepatic steatosis in chronic liver disease. Ultrasound Med Biol 2018;44:2223-2232.

32. Chalasani N, Younossi Z, Lavine JE, Charlton M, Cusi K, Rinella M, et al. The diagnosis and management of nonalcoholic fatty liver disease: practice guidance from the American Association for the Study of Liver Diseases. Hepatology 2018;67:328-357.

33. Borges VF, Diniz AL, Cotrim HP, Rocha HL, Andrade NB. Sonographic hepatorenal ratio: a noninvasive method to diagnose nonalcoholic steatosis. J Clin Ultrasound 2013;41:18-25.

34. Chauhan $A$, Sultan $L R$, Furth $E E$, Jones $L P$, Khungar V, Sehgal CM. Diagnostic accuracy of hepatorenal index in the detection and 
grading of hepatic steatosis. J Clin Ultrasound 2016;44:580-586.

35. Tsui PH, Ho MC, Tai DI, Lin YH, Wang CY, Ma HY. Acoustic structure quantification by using ultrasound Nakagami imaging for assessing liver fibrosis. Sci Rep 2016;6:33075.

36. Yamada H, Ebara M, Yamaguchi T, Okabe S, Fukuda H, Yoshikawa $M$, et al. A pilot approach for quantitative assessment of liver fibrosis using ultrasound: preliminary results in 79 cases. J Hepatol 2006;44:68-75.

37. Wu S, Tu R, Liang $X$. Patchy echogenicity of the liver in patients with chronic hepatitis B does not indicate poorer elasticity. Ultrasonography 2019;38:327-335.

38. Jeon SK, Lee JM, Joo I, Yoon JH, Lee DH, Lee JY, et al. Prospective evaluation of hepatic steatosis using ultrasound attenuation imaging in patients with chronic liver disease with magnetic resonance imaging proton density fat fraction as the reference standard. Ultrasound Med Biol 2019;45:1407-1416.

39. Tada T, Kumada T, Toyoda H, Yasuda S, Sone Y, Hashinokuchi S, et al. Liver stiffness does not affect ultrasound\&\#8208; guided attenuation coefficient measurement in the evaluation of hepatic steatosis. Hepatol Res 2020;50:190-198.

40. Castera L, Friedrich-Rust M, Loomba R. Noninvasive assessment of liver disease in patients with nonalcoholic fatty liver disease. Gastroenterology 2019;156:1264-1281. 